\section{A RANDOMIZED, PROSPECTIVE TRIAL OF STAPLED LUNG REDUCTION VERSUS LASER BULLECTOMY FOR DIFFUSE EMPHYSEMA}

Two procedures (laser bullectomy and lung reduction surgery with staples) are currently available for the surgical treatment of patients with diffuse emphysema. We compared the efficacy of these two surgical approaches in 72 patients, aged $67 \pm 7$ years (mean \pm standard deviation), who had diffuse emphysema scored as severe on computed tomography and severe fixed expiratory airflow obstruction. The patients were prospectively randomized to undergo either neodymium:yttrium aluminum garnet contact laser surgery $(n=33)$ or stapled lung reduction surgery $(n=39)$ by unilateral thoracoscopy. The operative mortalities were $0 \%$ and $2.5 \%$, respectively. No significant differences were noted between the groups $(p<$ 0.05 ) with respect to operating time, hospital days, or air leakage for more than 7 days. However, a delayed pneumothorax developed in six patients (18\%) who had laser treatment $(p=0.005)$. The operations eliminated dependency on supplemental oxygen in $52 \%$ of the laser group and $87.5 \%$ of the stapled lung reduction group $(p=0.02)$. The mean postoperative improvement in the forced expiratory volume in 1 second at 6 months was significantly greater for the patients undergoing the staple technique (32.9\% vs $13.4 \%, p=0.01$ ) than for the laser treatment group. (J THORAC CARDiovasc SuRG 1996;111:317-22)

Robert J. McKenna, Jr., MD (by invitation), Matthew Brenner, MD* (by invitation), Arthur F. Gelb, MD (by invitation), Michael Mullin, MD (by invitation), Narindar Singh, MD (by invitation), Harold Peters, MD (by invitation), John Panzera, RRT (by invitation), Jane Calmese, RN (by invitation), and Mark J. Schein, MD (by invitation), Orange, Lakewood, and Irvine, Calif.

Sponsored by Quentin R. Stiles, MD, Los Angeles, Calif.
A resurgence of interest has been noted in the surgical treatment for patients with diffuse emphysema. Forty years ago, Brantigan and Mueller ${ }^{1}$ performed multiple wedge resections for diffuse emphysema through a posterolateral thoracotomy

From the Lung Center, Chapman Medical Center, Orange, Calif; the Pulmonary (A.F.G.) and Radiology Departments (M.J.S.), Lakewood Regional Medical Center, Lakewood, Calif.; Pulmonary Division, University of California Irvine, Medical Center (M.B.), Irvine, Calif.; and Department of Thoracic Surgery, University of Southern California.

Read at the Seventy-fifth Annual Meeting of The American Association for Thoracic Surgery, Boston, Mass., April 23-26, 1995.

Address for reprints: Robert J. McKenna, Jr., MD, 1245 Wilshire Blvd., Suite 606, Los Angeles, CA 90017.

*Supported in part by Department of Education grant DEf603-91 ER61227 and National Institutes of Health grant R01192.

Copyright (c) 1996 by Mosby-Year Book, Inc.

$0022-5223 / 96 \$ 5.00+0 \quad \mathbf{1 2 / 6 / 7 0 3 0 0}$ incision. Although significant clinical improvement occurred in $75 \%$ of their patients, the mortality was $16 \%$, and the procedure never became popular. ${ }^{1}$

Recently, Wakabayashi and associates ${ }^{2}$ performed laser bullectomy on such patients via unilateral thoracoscopy. Short-term follow-up showed a 3-month mortality of $14 \%$ (3/22 patients) and a 36\% improvement in the forced expiratory volume in 1 second $\left(\mathrm{FEV}_{1}\right)^{2,3}$ The degree of improvement is artificially inflated by the inclusion of some patients with giant bullae.

In 1994, Cooper and coworkers ${ }^{4,5}$ rekindled interest in resectional treatment of diffuse emphysema via a median sternotomy with bovine pericardium to buttress the staples. They reported an $82 \%$ improvement in the $\mathrm{FEV}_{1}$ (mean follow-up 6.4 month) and improvement in dyspnea index, exercise tolerance, and quality of life in 20 patients.

This randomized, prospective trial was designed to compare the efficacy of these two surgical ap- 
Table I. Baseline comparison of patients in the laser and staple-treated surgical groups

\begin{tabular}{|c|c|c|c|}
\hline & Laser & Staple & $\begin{array}{c}p \\
\text { Value }\end{array}$ \\
\hline No. of patients & 33 & 39 & NS \\
\hline Male (No.) & 26 & 32 & NS \\
\hline Age $(y r)$ & $69 \pm 6$ & $66 \pm 8$ & NS \\
\hline $\mathrm{FEV}_{1}(\mathrm{~L})$ & $0.7 \pm 0.2$ & $0.7 \pm 0.2$ & NS \\
\hline FVC (L) & $2.1 \pm 0.7$ & $2.1 \pm 0.7$ & NS \\
\hline RV (L) & $5.1 \pm 1.1$ & $5.4 \pm 0.2$ & NS \\
\hline $\operatorname{TLC}(\mathrm{L})$ & $7.6 \pm 1.4$ & $7.9 \pm 1.3$ & NS \\
\hline DLCOSB $(\mathrm{ml} / \mathrm{min} / \mathrm{mm} \mathrm{Hg})$ & $5.4 \pm 3.0$ & $8.6 \pm 19$ & $\mathrm{NS}$ \\
\hline $\mathrm{PaO}_{2}(\mathrm{~mm} \mathrm{Hg})$ & $65 \pm 12$ & $66 \pm 12$ & NS \\
\hline $\mathrm{PaCO}_{2}(\mathrm{~mm} \mathrm{Hg})$ & $43 \pm 7$ & $44 \pm 8$ & NS \\
\hline SGaw $\left(\mathrm{L} / \mathrm{sec} / \mathrm{cm} \mathrm{H}_{2} \mathrm{O} / \mathrm{L}\right)$ & $0.03 \pm 0.01$ & $0.03 \pm 0.01$ & NS \\
\hline Smoking (pack-years) & $62 \pm 20$ & $68 \pm 24$ & NS \\
\hline CT emphysema score 8 & $60 \pm 21$ & $56 \pm 21$ & NS \\
\hline Dyspnea grade ${ }^{7}$ & $3.1 \pm 0.6$ & $3.1 \pm 0.7$ & NS \\
\hline Oxygen use (No. of patients) & 25 & 27 & NS \\
\hline
\end{tabular}

$\overline{F E V_{1}}$, Forced expiratory volume in 1 second; $F V C$, forced expiratory volume; $R V$, residual volume, $T L C$, total lung capacity; $D_{L} C O S B$, singlebreath diffusing capacity; $\mathrm{PaO}_{2}$, arterial oxygen tension; $\mathrm{PaCO}_{2}$, arterial carbon dioxide tension; $S G a w$, specific airway conductance; $C T$, computed tomography; NS, not significant. Data are presented as mean \pm standard deviation.

proaches. Because laser bullectomy is performed unilaterally by thoracoscopy, the stapled lung reduction technique was also performed unilaterally by thoracoscopy.

\section{Patients and methods}

Between June 1994 and November 1994, 72 patients aged $67 \pm 7$ years (mean \pm standard deviation [SD]) were randomized. All patients had severe symptoms with a substantial decrease in the dyspnea index and poor quality of life despite maximal medical management. Preoperatively, $49(68 \%)$ patients were receiving steroids and 52 (72\%) were using supplemental oxygen. No patients had been accepted for lung transplantation or had $\alpha_{1}$-antitrypsin deficiency. Chest roentgenograms showed hyperexpansion with flattening or inversion of the diaphragm. The heterogeneous pattern of emphysema on computed tomography (CT) was graded as severe and without isolated bullae larger than $5 \mathrm{~cm}$.

Contraindications included current cigarette smoking, age older than 75 years, carbon dioxide retention greater than $55 \mathrm{~mm} \mathrm{Hg}$, severe cardiac disease, history of cancer within the past 5 years, ventilator dependency, presence of a lung mass, or prior thoracic surgery. All patients who underwent surgery for emphysema were randomized into this study unless (1) they had previously undergone an operation on the contralateral lung for emphysema at another institution, (2) a lung mass was identified, or (3) a large $(>5 \mathrm{~cm})$, discrete bulla was present.

This protocol was approved by the Institutional Review Board at Chapman Medical Center. After informed consent was obtained, patients were blindly randomized to either neodymium:yttrium-aluminum-garnet (Nd:YAG) contact laser or resection of severe emphysematous areas of lung with staples and bovine pericardium. No patient refused randomization or crossed over to the other technique.

Baseline characteristics of the two surgical patient groups appear in Table I. All of the patients had severe fixed airflow limitation. The forced vital capacity (FVC) was $58 \% \pm 9 \%$ of predicted values (mean $\pm \mathrm{SD}$ ) and the $\mathrm{FEV}_{1}$ was $27 \% \pm 6 \%$ of predicted values (mean $\pm \mathrm{SD}$ ). There were no statistically significant differences between the two groups with respect to age, sex, lung function, arterial oxygen tension, arterial carbon dioxide tension, CT emphysema score, smoking, and need for daily oxygen use for an arterial oxygen tension of less than $55 \mathrm{~mm} \mathrm{Hg}$ at rest or during exercise. There was no significant difference between the laser and staple treatment groups $(p=$ NS) with respect to right versus left lung or upper versus lower prevalence of emphysema.

Pulmonary rehabilitation was not routinely done before the operation, but all patients began rehabilitation in the hospital after the operation. This included education regarding proper breathing techniques, anxiety control, stretching and strengthening exercises, and walking on the stairs and treadmill. The program was continued on an outpatient basis at Chapman Medical Center for 2 to 3 weeks after discharge. The vast majority of patients lived a long distance from our hospital, and they were eager to return home. They were strongly encouraged to join a Better Breathers' Club and rehabilitation program at home.

Operative technique. All patients underwent unilateral video-assisted thoracic surgery (VATS). They were paralyzed with pipecuronium and given general anesthesia with isoflurane. The procedure was done through a leftsided double-lumen tube (Mallincrodt Anesthesia, St. Louis, Mo.). The trocar and thoracoscope were placed through the tenth intercostal space in the posterior axillary line. Three additional 1 to $2 \mathrm{~cm}$ incisions were made for standard instruments, such as ring forceps.

The laser procedure was performed with a contact tip on the Nd:YAG laser at $10 \mathrm{~W}$. The entire pleural surface of the lung, including the fissures, was treated with the laser until maximal shrinkage was obtained. The less involved areas showed minimal shrinkage. The average number of joules per case was 22,598 (range 14,156 to 29,115 joules).

For the staple procedure, the worst areas of destroyed lung identified by the preoperative CT were resected. Ring forceps manipulated the lung into a $60 \mathrm{~mm}$ endoscopic stapler (ELC 60, Ethicon, Inc., Somerville, N.J.) with bovine pericardium (Peristrips, Biovascular, St. Paul, Minn.) to buttress the staples. The average number of staples fired per case was 9 (range 4 to 14). Approximately $20 \%$ to $30 \%$ of the lung was resected. Specimen weight ranged from 30 to $90 \mathrm{gm}$. Pleural tents and pleurodesis were not performed.

For both procedures, the chest was drained with two apically placed chest tubes. The patients routinely were taken to the intensive care unit for an overnight stay.

Lung function studies. All patients underwent studies of arterial blood gases and lung function that included static lung volumes measured by plethysmographic tech- 
niques, ${ }^{6}$ as well as timed spirometry and single-breath diffusing capacity in accordance with American Thoracic Society recommendations. ${ }^{7,8}$ Values were then compared with those predicted. ${ }^{9-11}$ All patients were considered to have fixed airflow limitation because the $\mathrm{FEV}_{1}$ after three inhalations of aerosolized albuterol $(670 \mu \mathrm{g})$ improved more than $12 \%$ or more than $200 \mathrm{ml}^{6}$ Maximum inspiratory and expiratory flow volume curves, thoracic gas volume, ${ }^{10}$ and airway resistance ${ }^{11}$ were measured in a plethysmograph (models 2800 and 6200, Sensormedics, Inc., Yorba Buena, Calif.) and compared with predicted values. ${ }^{10}$ The reciprocal of airway resistance ${ }^{10}$ is conductance; it was divided by the thoracic gas volume at which it was measured and specific conductance was calculated. Normal values are greater than $0.7 \mathrm{~L}$ per second per centimeter of water per liter. ${ }^{12}$ Residual volume was calculated by subtracting vital capacity from total lung capacity. Complete lung function studies were obtained before the operation and only timed spirometry and arterial blood gas studies were obtained 6 months after the operation.

Statistical methods. Group description statistics are expressed as mean \pm standard error of the mean unless otherwise specified. Differences between groups are compared by means of two-tailed paired $t$ tests with $p<0.05$ being considered significant. Pearson's $\chi^{2}$ statistics are used to determine differences in laser- versus stapletreated groups for categoric variables.

Clinical evaluation. Before the operation and 6 months after the operation, an MOS-36 quality-of-life questionnaire $^{12}$ and dyspnea index were scored to document lifestyle improvement and response to surgical intervention.

\section{Results}

The mean postoperative length of stay was $10.6 \pm$ 2.0 days (range 4 to 20 days) for the laser group and $13.7 \pm 1.9$ days (range 3-66 days) for the staple treatment group ( $p=\mathrm{NS}$ ). All chest tubes were removed before discharge, and in most cases the patients were discharged on the day that the chest tubes were removed. The chest tubes were in place for 9.8 days for the laser group and 13.2 days for the staple-treatment group ( $p=$ not significant). Prolonged air leak was primarily responsible for the longer length of stay in the stapletreatment group.

Mortality. No deaths occurred in the laser group and one death $(2.5 \%)$ in the staple-treatment group owing to a contralateral tension pneumothorax. No further deaths have occurred in the staple-treatment group, but three patients in the laser group have died since discharge from the hospital. One patient, whose clinical condition had improved, died of a self-inflicted gunshot wound 6 weeks after the operation. Another patient was clinically well but died 3 months later in his sleep. The condition of the third
Table II. Comparison of the complications for the two procedures

\begin{tabular}{lccc}
\hline & $\begin{array}{c}\text { Laser } \\
(n=33)\end{array}$ & $\begin{array}{c}\text { Staple } \\
(n=39)\end{array}$ & $\begin{array}{c}p \\
\text { Value }\end{array}$ \\
\hline Hospital days (mean $\pm \mathrm{SD})$ & $11 \pm 12$ & $13 \pm 11$ & $\mathrm{NS}$ \\
Air leak $>7$ days (No.) & $11(33 \%)$ & $19(48 \%)$ & $\mathrm{NS}$ \\
Death (No.) & 0 & $1(2.5 \%)$ & $\mathrm{NS}$ \\
Respiratory failure (No.) & 0 & $1(2.5 \%)$ & $\mathrm{NS}$ \\
Operating time (hr) & $2.1 \pm 0.7$ & $1.9 \pm 1.0$ & $\mathrm{NS}$ \\
Take back (No.) & $1(3 \%)$ & $1(2.5 \%)$ & $\mathrm{NS}$ \\
Ileus (No.) & $1(3 \%)$ & 0 & $\mathrm{NS}$ \\
Deep vein thrombosis (No.) & 0 & $1(2.5 \%)$ & $\mathrm{NS}$ \\
Delayed pneumothorax (No.) & $6(18 \%)$ & 0 & 0.005 \\
\hline
\end{tabular}

Respiratory failure is reintubation and use of mechanical ventilator, takeback is second thoracotomy for persistent air leaks, and pneumothorax is pneumothorax requiring chest tube and hospitalization. Data are presented as mean \pm standard deviation or percent incidence.

patient steadily deteriorated after laser bullectomy and he died of respiratory failure 3 months later.

Morbidity. The morbidity for the two procedures is shown in Table II. In each group, one patient underwent reoperation for closure of a persistent air leak. One patient in the staple-treatment group had a 48-day postoperative course complicated by respiratory failure when massive hyperexpansion that physiologically behaved like a tension pneumothorax developed in the contralateral lung. This decreased the cardiac index to $0.8 \mathrm{~L} / \mathrm{m}^{2}$ and the patient required separate ventilators for the right and left lungs. He ultimately made a full recovery, and 6 months later he has a $75 \%$ improvement in the $\mathrm{FEV}_{1}$ compared with the preoperative value. One patient in the laser group had massive intestinal air and an ileus that prolonged the hospital course to 12 days.

Delayed pneumothorax occurred 2 weeks to 4 months after discharge in six $(18 \%)$ patients who underwent the laser procedure. This occurrence resulted in significant clinical deterioration that lasted months before recovery. This complication did not occur in patients who underwent the staple procedure $(p=0.005)$.

Clinical status. The MOS-36 quality-of-life questionnaire showed significant improvement and dyspnea index improved by more than one grade in eight of the $33(24 \%)$ laser-treated patients compared with 26 of the $39(66 \%)$ patients treated with the staple technique $(p=0.003)$.

Oxygen use. Of the 25 patients who were dependent on supplemental oxygen before laser bullectomy, 13 (52\%) no longer require supplemental 
Table III. Results of various techniques for lung reduction surgery for diffuse emphysema

\begin{tabular}{lllcl} 
First author & Procedure & Method & $\begin{array}{c}\text { No. of } \\
\text { patients }\end{array}$ & $\begin{array}{c}F E V_{1} \\
(\%)\end{array}$ \\
\hline McKenna & YAG contact & Unilateral & 33 & 13.4 \\
Little $^{13}$ & YAG & Unilateral & 55 & 18 \\
Eugene $^{14}$ & YAG/KTP + & Unilateral & 28 & 34 \\
& Staples & & & \\
McKenna $_{\text {Cooper }}{ }^{4}$ & Staples & Unilateral & 39 & 33 \\
& Staples & Bilateral & 20 & 82 \\
\hline
\end{tabular}

The laser procedure was performed in the free beam mode unless otherwise stipulated. Method indicates whether the procedure was performed unilaterally or bilaterally.

oxygen 6 months after the procedure. Of the 27 patients who were receiving supplemental oxygen before stapled lung reduction, $22(87.5 \%)$ did not require supplemental oxygen 6 months after the procedure $(p=0.02)$.

Lung function (Table III). Pulmonary function test follow-up was obtained from 26 of 30 surviving laser patients $(87 \%)$ and 36 of 38 surviving stapletreatment patients $(95 \%)$ ). The six other patients refused to undergo follow-up pulmonary function tests. The mean improvement in $\mathrm{FEV}_{1}$ at 6 months was $0.09 \mathrm{~L}(13.4 \% \pm 5.5 \%)$ for the laser group and $0.22 \mathrm{~L}(32.9 \% \pm 4.8 \%)$ for the staple-treatment group $(p=0.01)$.

Six months after the operation, the mean increase in the FVC was $0.13 \mathrm{~L}(6 \% \pm 3 \%)$ for the laser bullectomy group and $0.35 \mathrm{~L}(21 \% \pm 6 \%)$ for the staple-treatment group ( $p=0.07)$. Only in the staple-treatment group was the improvement in the FEV 1 and the FVC from baseline significant ( $p$ $<0.006)$.

\section{Discussion}

Operations for emphysema are currently being performed unilaterally and bilaterally at various institutions, and the technique for the lung reduction operation may be either laser or stapling techniques, or a combination of the two. This study was specifically designed to compare the efficacy of the laser and stapling techniques. Because laser bullectomy is performed by unilateral thoracoscopy, the stapling technique was also performed by unilateral thoracoscopy.

This prospective, blindly randomized study demonstrates that patients in whom lung volume reduction surgery is done with staples and bovine pericardium have a lower morbidity (fewer delayed pneumothoraces), as well as greater improvement in oxygen independence, lung function, and overall lifestyle and dyspnea scale, than patients treated with the Nd:YAG contact laser.

Little information has been published regarding the efficacy of the various procedures for the surgical treatment of diffuse emphysema. Little and associates $^{13}$ reported a series of 55 patients with diffuse emphysema who underwent unilateral freebeam laser treatment with the Nd:YAG laser. The preoperative $\mathrm{FEV}_{1}$ improved from 0.74 to $0.85 \mathrm{~L}$ $(18 \%)$.

Eugene and coworkers ${ }^{14}$ reported the results of staples and either free-beam potassium-titanylphosphate or Nd:YAG laser therapy for 28 patients with diffuse emphysema. The $\mathrm{FEV}_{1}$ increased from 0.68 to $0.91 \mathrm{~L}(34 \%)$. There were no hospital deaths, but three patients died within 3 months $(11 \%)$. Subjective improvement occurred in 22 of 28 patients $(78.6 \%)$, and the preoperative oxygen dependence was eliminated in five of 23 (21\%) patients.

With a bilateral stapling technique, Cooper ${ }^{5}$ reported marked clinical improvement and a mean increase of $82 \%$ in the $\mathrm{FEV}_{1}$ and $27 \%$ in the FVC compared with baseline at 6 months in patients with lung function similar to that of the patients in the current study. In his 20 patients, Cooper reported no mortality, a mean hospital stay of 15 days, air leak for more than 7 days in 11 patients, and reexploration in four patients.

The results of the current study are compared with the results of these studies in Table III. The Nd:YAG laser without staples shows similar results with either a free beam (Little and coworkers ${ }^{13}$ ) or contact tip (current study). The combination of the staples and laser procedure (Eugene and colleagues ${ }^{14}$ ) provided the same improvement in pulmonary function achieved with staples alone (current study). Little's group reported a $20 \%$ incidence of delayed pneumothorax, which is similar to our $18 \%$ incidence. Delayed pneumothorax is not mentioned by Eugene and associates. The risk of additional morbidity (delayed pneumothorax) and the lack of additional benefit from the use of the laser has led us to abandon its use until a definite role for it can be identified.

Mechanisms for improved pulmonary function after operations for emphysema have been studied. Rogers, ${ }^{15}$ Gelb, ${ }^{16}$ and their colleagues have found that resection of large bulla in isolated bullous disease and bullous emphysema resulted in improvement in expiratory airflow and airway conductance that could be accounted for by the improve- 
ment in elastic recoil. Similar mechanisms may explain the improved lung function after lung reduction operations for generalized emphysema.

Most patients who are candidates for lung reduction surgery have a thin superficial layer of better lung tissue and clusters of 1 to $2 \mathrm{~cm}$ bullae (severe emphysematous change) located deeper within the lobe. The laser is excellent for shrinking superficial areas of emphysema but does not have much effect on lung tissue with minimal emphysematous change. The laser does not penetrate to the deeper emphysematous areas within the lobe. The staple procedure may be more effective because aggressive resection of emphysematous areas can provide greater lung reduction than the shrinkage that results from the use of the laser. This allows for the greatest potential improvement in lung elastic recoil.

On the basis of the results of this study and previous studies, ${ }^{1-5}$ our current procedure of choice for diffuse emphysema is bilateral lung reduction surgery with staples. We have used the median sternotomy, but thoracoscopy continues to be the primary method of performing these lung reduction procedures at our institution. The lateral approach (with thoracoscopy) provides better access than the median sternotomy if the area of emphysema targeted for resection is located posteriorly and better access for lysis of posteriorly located adhesions (which have been found in approximately $30 \%$ of our patients). The majority of the operations can probably be performed equally well through either approach.

The surgical treatment of emphysema is palliative, and patients are expected to eventually return to their preoperative level of dyspnea if they survive long enough. Numerous patients in this study are no longer significantly limited by dyspnea. This fact raises the question of whether this procedure should be performed unilaterally or bilaterally. If preoperative testing could identify which patients can be adequately treated by a unilateral operation, perhaps the other side should be saved for the future in case of subsequent development of new-onset dyspnea. Long-term follow-up will answer this question.

In conclusion, our results demonstrate a lower morbidity (fewer delayed air leaks) and a greater overall improvement in clinical status for patients with generalized emphysema after surgery with staples and bovine pericardium than with the Nd:YAG contact laser.

\section{REFERENCES}

1. Brantigan OC, Mueller E. Surgical treatment of pulmonary emphysemá. Am Surg 1957;23:789-804.

2. Wakabayashi A, Brenner M, Kayaleh RA, et al. Thoracoscopic carbon dioxide laser treatment of bullous emphysema. Lancet 1991;337:881-3.

3. Brenner M, Kayaleh RA, Milne EN, et al. Thoracoscopic laser ablation of pulmonary bullae. J THORAC Cardiovasc Surg 1994;107:883-90.

4. Cooper JD, Trulock EP, Triantafillou AN, et al. Bilateral pneumectomy (volume reduction) for chronic obstructive pulmonary disease. J THORAC Cardiovasc Surg 1995;109:106-19.

5. Cooper JD. Technique to reduce air leaks after resection of emphysematous lung. Ann Thorac Surg 1994:57:1038-9.

6. American Thoracic Society. Lung function testing: selection of reference values and interpretative strategies. Am Rev Respir Dis 1991;144:1202-18.

7. American Thoracic Society. Single breath carbon monoxide diffusing capacity (transfer factor): recommendation for a standard technique. Am Rev Respir Dis 1987;136:1307-29.

8. Goldman HI, Becklake MR. Respiratory function tests: normal values at median altitudes and the prediction of normal results. Am Rev Tuberc Pulm Dis 1971;103:57-67.

9. Gaensler EA, Smith AA. Attachment for automated single breath diffusing capacity measurement. Chest 1973;63:136-45.

10. DuBois AB, Botelho SY, Comroe JH Jr. A new method for measuring airway resistance in man using a body plethysmograph: values in normal subjects and in patients with respiratory disease. J Clin Invest 1956;35:327-35.

11. Pelzer AM, Thompson ML. Effect of age, sex, stature and smoking habit on human airway conductance. $\mathbf{J}$ Appl Physiol 1966;21:469-76.

12. McHorney CA, Ware JE, Raczek AE. The MOS 36-item short-form health survey (SF-36). II. Psychometric and clinical tests of validity in measuring physical and mental health constructs. Med Care 1993;31:247-63.

13. Little AG, Swain JA, Nino JJ, Prabhu RD, Schlachter MD, Barcia TC. Reduction pneumoplasty for emphysema: early results. Ann Surg 1995;222:365-74.

14. Eugene J, Ott RA, Gogia HS, Dos Santos C, Zeit R, Kayaleh RA. Video-thoracic surgery for treatment of end-stage bullous emphysema and chronic obstructive pulmonary disease. Am Surg 1995;61:934-6.

15. Rogers RM, DuBois AB, Blakemore WS. Effects of removal of bullae on airway conductance and conductance volume ratios. J Clin Invest 1968;47:2569-79.

16. Gelb AF, Gold WM, Nadel JA. Mechanisms limiting airflow in bullous disease. Am Rev Respir Dis 1973; 107:571-8. 


\section{Discussion}

Dr. Alec Patterson (St. Louis, Mo.). You have demonstrated that laser therapy is a less satisfactory option in patients with diffuse emphysema. We had come to that conclusion ourselves some time ago. I think that there is a controversy as to whether patients should undergo unilateral or bilateral procedures. I am certain that there is room for controversy regarding whether a thoracoscopic or an open approach is preferable. Inasmuch as you have experience with both of those approaches, I would appreciate your comments on that matter.

In our patients in whom we have at least 6 months follow-up, there is a significant improvement in $\mathrm{FEV}_{1}$, about a $68 \%$ increase on average, and a decrease of $35 \%$ in residual volume. Most of the patients have demonstrated a significant improvement in gas exchange. $\mathrm{Pa}$ tients have for the most part been free of supplemental oxygen, although most required oxygen at the time of their assessment $-90 \%$ with exercise before the operation and $27 \%$ with exercise after the operation. All of these patients underwent bilateral volume reduction conducted through a median sternotomy.

These results are achieved with exactly the same mortality, hospital stay, and morbidity. Given these results, I would like your opinion on the benefit of a unilateral operation.

You stated that patients who had excessive carbon dioxide tension or who were hypercarbic were excluded from your series. Is there a clinical circumstance or a point at which you would not recommend surgery purely on the basis of carbon dioxide tension?

Dr. McKenna. First the question regarding use of a unilateral versus bilateral approach: At this point, our experience with lung reduction surgery comprises about 225 patients. About 100 of those patients have had bilateral operations. Of those, 20 had median sternotomies and the rest had bilateral thoracoscopic approaches.

Some patients who have had unilateral procedures are playing golf and doing everything that they want. Because they are not limited at all physically, I do not know how much more benefit they could accrue from the bilateral procedure. I do not know how long the benefits of the unilateral procedure will last compared with those of the bilateral procedure. We will be observing our patients who have had unilateral and bilateral procedures to determine whether it is better for the patients to undergo a bilateral procedure or sequential unilateral procedures. Long-term follow-up is needed to address that issue.

Your second question concerned the issue of thoracoscopy versus median sternotomy. As I mentioned, I have done 20 median sternotomies. There is definitely a role for each approach, but the majority of surgeons will feel more comfortable doing the procedure via a median sternotomy. I choose that approach if there is such a large disparity in the pulmonary function between the two sides that I do not think I can collapse the lung long enough for a thoracoscopic procedure.

In certain situations I think a lateral approach is better than an anterior approach for these operations. About a third of our patients have adhesions that are primarily found apically, posteriorly, and on the left side by the aorta. I believe lysis of those adhesions can be accomplished more easily with thoracoscopy in a lateral approach than through a median sternotomy. The lateral approach also allows better access for resection of posteriorly located bullae. For those reasons, I tend to use the thoracoscopic approach in most cases. I believe the majority of operations can be done either way.

I have not routinely required preoperative pulmonary rehabilitation, but rather have used it selectively in patients whose condition deteriorated in recent months or in patients who retain carbon dioxide.

Dr. Claude Deschamps (Rochester, Minn.). Do you use postoperative pulmonary rehabilitation when you use it before the operation? Do you use the CT scan as a criterion for excluding patients from this type of treatment?

Dr. McKenna: As I mentioned, I do not routinely use pulmonary rehabilitation before the operation. All patients undergo pulmonary rehabilitation after the operation in the hospital. A large percentage of our patients are from around the country, and I insist that they stay in the area for 2 to 3 weeks for rehabilitation until they are clinically ready to go back home. We strongly encourage the patients to continue rehabilitation at home.

Dr. Deschamps. Do you use the homogeneity criterion on the CT scan?

Dr. McKenna. The CT scan is my most important selection criterion for these patients. We have examined patients who have diffuse disease, upper lobe disease predominantly, and lower lobe disease. They do not necessarily have $\alpha_{1}$-antitrypsin deficiency. With the bilateral approach that we routinely use, the average improvement in the $\mathrm{FEV}_{1}$ for the patients with upper lobe disease has been $83 \%$; for patients with diffuse disease, $53 \%$; and for patients with lower lobe disease, only $30 \%$. Only half of the third group has any improvement.

Currently I select patients with heterogeneous emphysema on thin-cut CT scans; I use the ventilation/perfusion scan to confirm that functionally their lungs behave the same way that CT scan suggests they will.

We are evaluating elastic recoil in all patients both before and after the operation, and we, too, have believed that patients who have a high pressure, more than $15 \mathrm{~mm}$ $\mathrm{Hg}$, are not candidates for the operation. 